\title{
TEXTUAL ERROR MEANING IN ENGLISH TO INDONESIAN TRANSLATION OF EFL CLASS
}

\author{
Dedy Subandowo ${ }^{1}$, Eva Faliyanti ${ }^{2}$, Yuli Triatmi ${ }^{3}$ \\ Muhammadiyah University of Metro, Lampung, Indonesia \\ dedy.ummetro@yahoo.com,
}

\begin{abstract}
This research addresses on textual error meaning of English to Indonesian translation in EFL class. This study is aimed to describe the frequency of error distribution and to investigate the dominant errors made by students in English to Indonesian Translation. The subject of this research is the fourth-semester English education students at the Muhammadiyah University of Metro. There are 37 students used as the total sampling technique to get the sample. To collect the data, the writer used a test as the instrument, i.e. an essay test. The collected data are analyzed by means of error analysis which starts from collecting the data, identifying the errors from the answer sheet, classifying and describing of errors on each type and finally tabulating the students' errors. The finding shows that frequency of errors made by the student in translating English into Indonesian can be classified into $73.43 \%$ of verb, $85 \%$ of noun, $52.7 \%$ of adverb and $100 \%$ of conjunction. On the other hand, the common error in translating textual meaning occurs in conjunction class which the average is $100 \%$. It is the biggest percentage of all the errors which is automatically be the dominant error of textual meaning in this research.
\end{abstract}

Keywords: textual error meaning, English-Indonesian translation,

\section{INTRODUCTION}

Translation is one of the language components that should be learned by the English students generally (Ismail, 2016). Translation is the process of putting something written or spoken in a different language (Manser, 2004). In order to achieve equivalent target text, the characteristics of the source and target language are crucial to take into account. Since each language has its own characteristics, English and Indonesian are different in terms of characteristics (Apriyanti, 2016). Therefore, translator needs to understand when and how to use these characteristics in translating. Hatim \& Munday (2004) define translation as "the process of transferring a written text from a source language (SL) to target language (TL)".

By learning translation, they can translate the English text or sentences into Indonesian or vice versa. Textual meaning is a text or words which have more than one meaning. First of all, convey the "textual meaning". According to Eggins in (Hawalia, 2014) textual meaning, therefore "refers to the way the text is organized as a piece of writing (or speech)". The textual meaning has similarity with semantics because they explain about the meaning in the text or language. Translation is the process of putting something written or spoken into a different language. As students, they should be able to translate English into Indonesian 
well (Rahayu, 2015). The reason behind this situation is because of students' ability to understand complex sentence translation level in paragraphs is still quite difficult and unable to translate it well. The students or translator must master the language pairs, the source language, and the target language (Subandowo, 2017). But, in the fact, most of them are difficult to translate English into Indonesian well and make errors when they translate in the translation subject.

In this study, the writer took article from Jakarta Post that will be given by test, specifically the writer choose education article it is because this research is related to education. The education article that will be taken is about "Minister sees Germany, Switzerland as models for vocational schools", published on Thursday, January 26, 2017. The researcher conducted the pre-survey to find out students ability in translating text English into Indonesian. From the pre-survey, the writer assumes that students make common errors in translating English into Indonesian, specifically in textual meaning. The phenomena can be seen from the symptoms below, some of the students' difficulties to find appropriate word and meaning in translating English into Indonesian also some students do not understand about the content of the material.

\section{The Definition of Error}

In this study, the writer used some theories to support this research. According Ellis \& Gary (2005) errors occur because of gaps in the learner's English knowledge whereas error occurs when the learner has not yet learned how to master a certain grammatical form. Otherwise Brown (1994) and also Connor (1996) who categorized learner language errors into two types: interlingual and intralingual. Interlingual errors are those resulting from the influence the learner's native language or external factors, whereas intralingual errors are the ones resulting from the target language system itself or internal factors (Fauziati, 2017). Furthermore, error analysis is the study of learner's errors that can be observed, analyzed, and classified to reveal something of the system operating within the learner, led to a surge of the study of learner's errors (Durrani, 2018) and (Brown, 2000).

\section{Translation}

Translation is the process of putting something written or spoken into the different language (Manser, 2004). Otherwise, translation has its holistic moment as well as its more obvious atomistic aspect. Hatim \& Munday (2004) define translation as "the process of transferring a written text from a source language (SL) to target language (TL)". While Olk (2003) said, translation is an activity that needs substantial cultural knowledge. According to Eggins in (Hawalia, 2014), and (Yuliasri, 2016) textual meaning, therefore "refers to the way the text is organized as a piece of writing (or speech)". In other views, Nababan (2003) said that textual meaning is related to the context. In addition, translation is distinguished from translating which means the process of transferring source text into target text which involves translators and the psychological and intellectual functioning of their mind (Apriyanti, 2016). In summary, the nature of the translation is a translation result involving the process of transferring the source language to the target language. It was aimed at transforming the meaning of the text is inaccessible to one and can be understood in target language 
(Mujiyanto, 2017). The nature of the translation includes the process of transfer of meaning, understanding of the type of translation, and the translation component. It is a matter of finding the appropriate clearing and the appropriate language in which and form which the text speaks (Ashton, 2014).

\section{METHOD}

The researcher design applied in this study is descriptive analysis. According to (Arikunto, 2006) (Richard \& Schmidt, 2002) descriptive research studies are designed to obtain information concerning the status of phenomena. This research is qualitative research which concerned with developing explanations of social phenomena (Hancock, Ockleford, \& Windridge, 2009) (Sandelowski, 2000) (Cochran, 2002).

A population is a group of objects or subjects that have specific qualities and characteristics defined by the researchers to learn and then be concluded (Sugiyono, 2010). The population in this research is from all students English education program Muhammadiyah University of the metro, especially at the fourth semester. (Notoatmodjo, 2005) the sample is part of a population that represents the population that will be taken. The sample in this study is the student of the fourth semester of English education program of Muhammadiyah University of Metro, that there are at least 37 students. Sampling is a way to take a sample which really fits into the overall object of research (Nursalam, 2003). In this study, the writer used a total sampling technique to take the sample. Total sampling is a sampling technique that the count of a sample as same as population (Sugiyono, 2010).

In this research, the writer chooses a test as the instrument of collecting data. A test is a systematic procedure of observing one's behavior and describing it with the aid of numerical device and category system (Syakur, 1999). In this research, the researcher used an essay test to test the students' ability in translate English into Indonesian.

In processing the data, the first step was identified of any deviations in translation error English into Indonesian text which possibly could be found in the students' translation answer sheet. The next step was classified them into types of errors. A comparison between the original forms made by the students and the reconstruction of those forms in the target language indicates to which type of error belongs. The classification of error was done based on textual meaning. The last step was counted and tabulated, and the number of errors is presented in the form of the percentage.

\section{RESULTS AND DISCUSSION}

The finding of the research showed that there are eleven words and phrases of any possible properties of translation errors in students' textual meaning can be seen in the following table.

Table 1: The Sample of Textual Meaning Error in English into Indonesian Translation

\begin{tabular}{|l|c|c|c|c|}
\hline No & $\begin{array}{c}\text { The } \\
\text { Translation } \\
\text { Test }\end{array}$ & \multicolumn{2}{|c|}{ The Result Test Data } & $\begin{array}{c}\text { Identification } \\
\text { of Students }\end{array}$ \\
\cline { 2 - 6 } & $\begin{array}{c}\text { Source } \\
\text { Language }\end{array}$ & Key answer & Students' answer & $\begin{array}{c}\text { Students' } \\
\text { Number }\end{array}$ \\
\hline 1. & Minister sees & Menteri & $\bullet$ Menteri melihat Jerman, & $\bullet 01,02,03,04$, \\
\hline
\end{tabular}




\begin{tabular}{|c|c|c|c|c|}
\hline & $\begin{array}{l}\text { Germany, } \\
\text { Switzerland as } \\
\text { models for } \\
\text { vocational } \\
\text { schools }\end{array}$ & \begin{tabular}{lr}
\multicolumn{2}{l}{ Memandang } \\
Jerman, & Swiss \\
Sebagai & Contoh \\
Untuk & Sekolah \\
Kejuruan &
\end{tabular} & $\begin{array}{l}\text { Switzerland sebagai contoh } \\
\text { sekolah kejuruan } \\
\\
\text { - Menteri menunjuk Jerman, } \\
\text { Switzerland sebagai model } \\
\text { untuk sekolah kejuruan } \\
\text { - Menteri mengamati Negara } \\
\text { Jerman, Switzerland } \\
\text { sebagai model untuk } \\
\text { sekolah menengah kejuruan }\end{array}$ & $\begin{array}{l}06,10,13,14, \\
16,17,24,25, \\
27,28,30,31, \\
32,33,34,36, \\
37, \\
\text { - } 08,09,18,19, \\
21,22,23,29, \\
35 \\
-07\end{array}$ \\
\hline 2. & $\begin{array}{l}\text { Industry } \\
\text { Minister } \\
\text { Airlangga } \\
\text { Hartarto has } \\
\text { urged the } \\
\text { country to use } \\
\text { developed } \\
\text { countries like } \\
\text { Germany or } \\
\text { Switzerland as } \\
\text { examples in } \\
\text { developing } \\
\text { vocational } \\
\text { schools as an } \\
\text { inseparable } \\
\text { part of the } \\
\text { industrializatio } \\
\text { n process. }\end{array}$ & 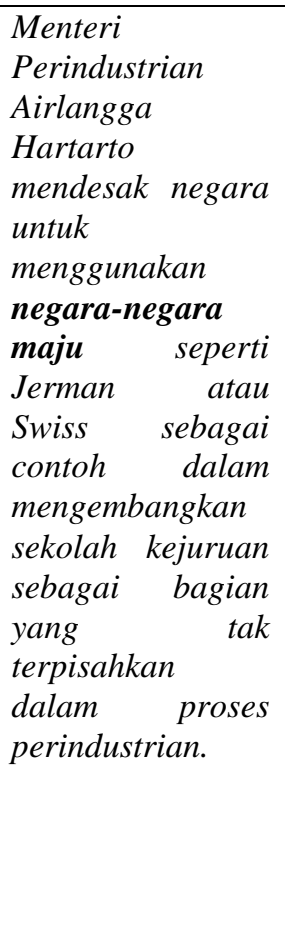 & $\begin{array}{l}\text { - Menteri industry Airlangga } \\
\text { Hartanto mendesak Negara } \\
\text { untuk menggunakan } \\
\text { Negara-negara } \\
\text { berkembang seperti Negara } \\
\text { Jerman atau Switzerland } \\
\text { sebagai contoh dalam } \\
\text { mengembangkan sekolah- } \\
\text { sekolah kejuruan sebagai } \\
\text { suatu bagian dari proses } \\
\text { perindustrian. } \\
\text { - } \\
\text { Henteri industry Airlangga } \\
\text { Negartanto telah mendesak } \\
\text { perkembangan menggunakan } \\
\text { negara seperti Jerman atau } \\
\text { Switzerland sebagai contoh } \\
\text { dalam perkembangan } \\
\text { sekola-sekolah kejuruan } \\
\text { sebagai suatu bagian yang } \\
\text { tidak dapat dipisahkan dari } \\
\text { proses peerindustrian. }\end{array}$ & $\begin{array}{l}-03,04,05,06, \\
07,11,12,13, \\
16,31,33,34, \\
35,\end{array}$ \\
\hline
\end{tabular}

Table 2: The Sample of Textual Meaning Error in English into Indonesian Translation

\begin{tabular}{|c|c|c|c|c|}
\hline \multirow[t]{2}{*}{ No } & & \multicolumn{2}{|c|}{ The Result Test Data } & \multirow{2}{*}{$\begin{array}{c}\begin{array}{c}\text { Identification } \\
\text { of Students }\end{array} \\
\begin{array}{c}\text { Students' } \\
\text { Number }\end{array} \\
\end{array}$} \\
\hline & $\begin{array}{c}\text { Source } \\
\text { Language }\end{array}$ & Key answer & Students' answer & \\
\hline & & & $\begin{array}{l}\text { - Menteri industry Airlangga } \\
\text { Hartanto telah mendesak } \\
\text { Negara untuk menggunakan } \\
\text { pengembangan Negara } \\
\text { seperti Jerman atau } \\
\text { Switzerland sebagai contoh } \\
\text { dalam perkembangan } \\
\text { sekolah-sekolah kejuruan } \\
\text { sebagai suatu bagian yang }\end{array}$ & - 15,26 \\
\hline
\end{tabular}


Journal of English Teaching Adi Buana, Vol. 04 No. 01, April 2019

\begin{tabular}{|c|c|c|c|c|}
\hline & & & $\begin{array}{l}\text { tidak dapat dipisahkan } \\
\text { proses perindustrian }\end{array}$ & \\
\hline 3. & $\begin{array}{l}\text { If we take a } \\
\text { look at } \\
\text { Germany or } \\
\text { Switzerland, } \\
\text { 16-year-old } \\
\text { kids start } \\
\text { getting } \\
\text { involved.... }\end{array}$ & $\begin{array}{l}\text { Apabila kita } \\
\text { melihat Jerman } \\
\text { atau Swiss, anak- } \\
\text { anak berusia } 16 \\
\text { tahun } \quad \text { mulai } \\
\text { terlibat .... }\end{array}$ & 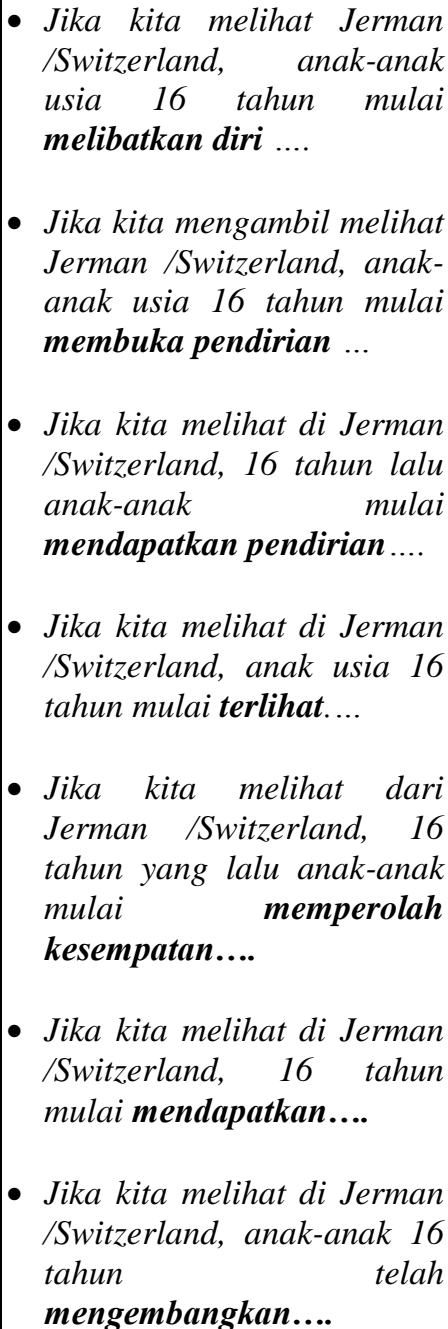 & $\begin{array}{l}-03,04,06,12, \\
13,31,33,34 \\
-05\end{array}$ \\
\hline
\end{tabular}

Table 3: The Sample of Textual Meaning Error in English into Indonesian Translation

\begin{tabular}{|c|c|c|c|c|}
\hline \multirow[t]{2}{*}{ No } & \multirow{2}{*}{$\begin{array}{c}\text { The } \\
\text { Translation } \\
\text { Test } \\
\text { Source } \\
\text { Language } \\
\end{array}$} & \multicolumn{2}{|c|}{ The Result Test Data } & \multirow{2}{*}{\begin{tabular}{|c|}
$\begin{array}{c}\text { Identification } \\
\text { of Students }\end{array}$ \\
$\begin{array}{c}\text { Students' } \\
\text { Number }\end{array}$ \\
\end{tabular}} \\
\hline & & Key answer & Students' answer & \\
\hline & & & $\begin{array}{l}\text { - Jika kita lihat di Negara } \\
\text { Jerman /Switzerland, anak- } \\
\text { anak pada usia } 16 \text { tahun } \\
\text { mulai dilibatkan.... }\end{array}$ & - 30,32 \\
\hline 4. & $\begin{array}{l}\text {....in the } \\
\text { industrializatio } \\
n \text { process by } \\
\text { being invited } \\
\text { to work as } \\
\text { interns in real } \\
\text { factories }\end{array}$ & $\begin{array}{l}\text {....dalam proses } \\
\text { industrialisasi } \\
\text { yang diundang } \\
\text { untuk bekerja } \\
\text { sebagai karyawan } \\
\text { magang di } \\
\text { pabrik-pabrik } \\
\text { yang sebenarnya }\end{array}$ & \begin{tabular}{|lr}
-...didalam & proses \\
perindustrian & bentuk yang \\
diundang & untuk bekerja \\
sebagai & karyawan \\
perusahaan & \\
& \\
&
\end{tabular} & $\begin{array}{l}-01,02,03,04, \\
06,07,11,12, \\
13,14,16,17, \\
22,23,24,25, \\
27,30,31,32, \\
33,34,35,36, \\
37 \\
-15\end{array}$ \\
\hline
\end{tabular}




\begin{tabular}{|c|c|c|c|c|}
\hline & & & $\begin{array}{l}\text { - ...di proses pengindustrian } \\
\text { menjadi mengajak untuk } \\
\text { bekerja sebagai pekerja } \\
\text { dipabrikyang sesungguhnya } \\
\text { - ...proses industri yang } \\
\text { digunakan untuk bekerja } \\
\text { sebagai factor-faktor yang } \\
\text { nyata } \\
\text {-..dalam proses } \\
\text { perindustrian dalam bentuk } \\
\text { undangan untuk bekerja } \\
\text { sebagaidokter rumah sakit }\end{array}$ & $\begin{array}{l}\text { - } 20 \\
\\
\\
\text { - } 08,09,10,18, \\
19,21,26,28, \\
29\end{array}$ \\
\hline 5. & $\begin{array}{l}\text {...in the } \\
\text { industrializatio } \\
\mathrm{n} \text { process by } \\
\text { being invited } \\
\text { to work as } \\
\text { interns in real } \\
\text { factories }\end{array}$ & $\begin{array}{l}\text {...dalam proses } \\
\text { industrialisasi } \\
\text { yang diundang } \\
\text { untuk bekerja } \\
\text { sebagai karyawan } \\
\text { magang di } \\
\text { pabrik-pabrik } \\
\text { yang sebenarnya }\end{array}$ & $\begin{array}{l}\text { - ...di proses pengindustrian } \\
\text { menjadi mengajak untuk } \\
\text { bekerja sebagai pekerja } \\
\text { dipabrik yang sesungguhnya } \\
\text { - ...dalam proses } \\
\text { perindustrian dalam bentuk } \\
\text { undangan untuk bekerja } \\
\text { sebagai dokter rumah sakit } \\
\text {...dalam proses } \\
\text { perindustrian dalam bentuk } \\
\text { undangan untuk magang } \\
\text { dipabrik yang nyata. }\end{array}$ & - 15 \\
\hline
\end{tabular}

Table 4: The Sample of Textual Meaning Error in English into Indonesian Translation

\begin{tabular}{|c|c|c|c|c|}
\hline \multirow[t]{2}{*}{ No } & & \multicolumn{2}{|c|}{ The Result Test Data } & Identification \\
\hline & $\begin{array}{c}\text { Source } \\
\text { Language }\end{array}$ & Key answer & Students' answer & $\begin{array}{l}\text { Students' } \\
\text { Number }\end{array}$ \\
\hline 6. & $\begin{array}{l}\text { As a result, by } \\
\text { the time they } \\
\text { reach } 19 \\
\text { they're already } \\
\text { prepared [to } \\
\text { enter the } \\
\text { workforce] }\end{array}$ & $\begin{array}{lr}\text { Alhasil, pada saat } \\
\text { mereka menginjak } \\
\text { usia 19 } & \text { tahun } \\
\text { mereka } & \text { sudah } \\
\text { siap } & \text { [untuk } \\
\text { menjadi } & \text { tenaga } \\
\text { kerja] } & \end{array}$ & $\begin{array}{l}\text { - Sebagai hasil, pada saat } \\
\text { mereka mencapai } 19 \text { tahun } \\
\text { umur mereka siap } \\
\text { mempersiapkan } r \text { untuk } \\
\text { masuk menjadi tenaga } \\
\text { kerja. } \\
\text { - Hasilnya, dari waktu ke } \\
\text { waktu mereka mencapai } \\
\text { usia } 19 \text { tahun mereka sudah } \\
\text { siap untuk memasuki dunia } \\
\text { kerja. } \\
\text { - Sebagai hasil, setiap waktu } \\
\text { mereka dapat menjangkau } \\
\text { dalam umur } 19 \text { tahun untuk } \\
\text { menyiapkannyar masuk } \\
\text { kepenguatan kerja. } \\
\text { - Sebagai hasil, diwaktu } \\
\text { mereka mencapai } 19 \text { tahun }\end{array}$ & $\begin{array}{l}\text { - } 01,14,16,17, \\
24,25,27,36, \\
37 \\
\\
\text { - } 03,15,18,19, \\
21,23,29,30, \\
32,34 \\
\text { - } 28 \\
\text { - } 22\end{array}$ \\
\hline
\end{tabular}




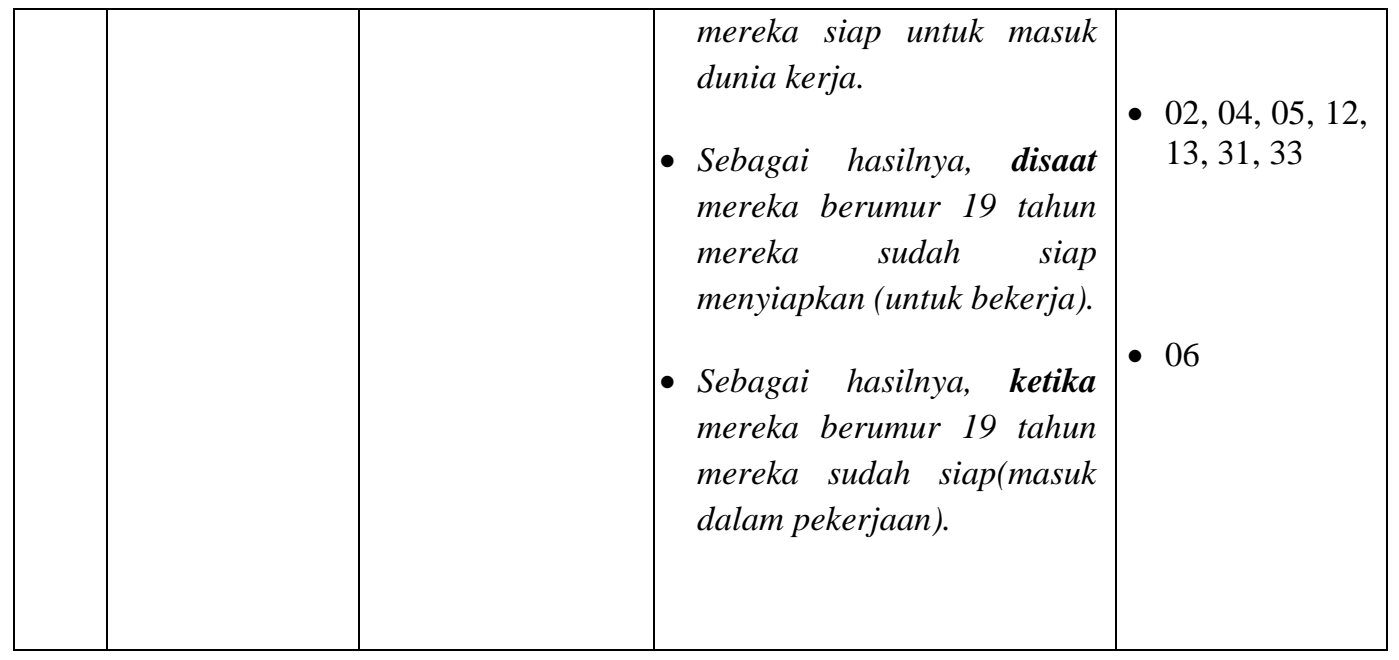

Table 5: The Sample of Textual Meaning Error in English into Indonesian Translation

\begin{tabular}{|c|c|c|c|c|}
\hline \multirow[t]{2}{*}{ No } & \multirow{2}{*}{$\begin{array}{c}\text { The } \\
\text { Translation } \\
\text { Test } \\
\text { Source } \\
\text { Language } \\
\end{array}$} & \multicolumn{2}{|c|}{ The Result Test Data } & \multirow{2}{*}{\begin{tabular}{|c|}
$\begin{array}{c}\text { Identification } \\
\text { of Students }\end{array}$ \\
$\begin{array}{c}\text { Students' } \\
\text { Number }\end{array}$ \\
\end{tabular}} \\
\hline & & Key answer & Students' answer & \\
\hline 7. & $\begin{array}{l}\text { As a result, by } \\
\text { the time they } \\
\text { reach } 19 \\
\text { they're already } \\
\text { prepared [to } \\
\text { enter the } \\
\text { workforce] }\end{array}$ & $\begin{array}{l}\text { Alhasil, pada saat } \\
\text { mereka } \\
\text { menginjak usia } \\
19 \text { tahun mereka } \\
\text { sudah siap [untuk } \\
\text { menjadi tenaga } \\
\text { kerja] }\end{array}$ & $\begin{array}{l}\text { - Sebagai hasil, pada saat } \\
\text { mereka mencapai } 19 \text { tahun } \\
\text { umur merekar siap } \\
\text { mempersiapkan untuk } \\
\text { masuk menjadi tenaga } \\
\text { kerja. } \\
\\
\text { - Sebagai hasil, setiap waktu } \\
\text { mereka dapat menjangkau } \\
19 \text { tahun untuk menyiapkan } \\
\text { (untuk masuk kepenguatan } \\
\text { kerja) } \\
\text { - Sebagai hasil, dari waktu } \\
\text { mereka jangkauan 19 tahun } \\
\text { untuk menyiapkan (untuk } \\
\text { masuk kedunia kerja) }\end{array}$ & $\begin{array}{l}-01,02,03,08, \\
09,11,14,17, \\
19,20,21,22, \\
23,24,25,27, \\
30,32,34,35, \\
36,37 \\
-18,28\end{array}$ \\
\hline .8 . & $\begin{array}{l}\text { As a result, by } \\
\text { the time they } \\
\text { reach } 19 \\
\text { they're already } \\
\text { prepared [to } \\
\text { enter the } \\
\text { workforce] }\end{array}$ & $\begin{array}{lr}\text { Alhasil, pada saat } \\
\text { mereka menginjak } \\
\text { usia 19 } 19 \text { tahun } \\
\text { mereka } & \text { sudah } \\
\text { siap } & \text { [untuk } \\
\text { menjadi } & \text { tenaga } \\
\text { kerja] } & \end{array}$ & $\begin{array}{l}\text { - Sebagai hasil, pada saat } \\
\text { mereka mencapai } 19 \text { tahun } \\
\text { umur mereka siap } \\
\text { mempersiapkan (untuk } \\
\text { menjadi tenaga kerja). } \\
\text { - Sebagai hasil, dari waktu } \\
\text { mereka mencapai } 19 \text { tahun } \\
\text { mereka menyiapkan (untuk } \\
\text { masuk kedunia kerja) }\end{array}$ & $\begin{array}{l}-02,03,07,08, \\
09,11,14,16, \\
17,21,22,25, \\
27,29,30,32, \\
34,35,36,37 \\
\\
-04,05,06,10, \\
12,13,15,19, \\
26,31,33\end{array}$ \\
\hline
\end{tabular}




\begin{tabular}{|l|l|l|}
\hline & $\begin{array}{l}\text { mereka berusia } 19 \text { tahun } \\
\text { mereka sudah siap (masuk } \\
\text { dalam pekerjaan). } \\
\text { - Sebagai hasil, setiap waktu } \\
\text { mereka dapat menjangkau } \\
\text { dalam umur 19 tahun untuk } \\
\text { menyiapkannya (masuk } \\
\text { kepenguatan kerja). }\end{array}$ \\
\hline
\end{tabular}

Table 6: the Sample of Textual Meaning Error in English into Indonesian Translation

\begin{tabular}{|c|c|c|c|c|}
\hline \multirow[t]{2}{*}{ No } & & \multicolumn{2}{|c|}{ The Result Test Data } & \multirow{2}{*}{$\begin{array}{c}\begin{array}{c}\text { Identification } \\
\text { of Students }\end{array} \\
\begin{array}{c}\text { Students' } \\
\text { Number }\end{array}\end{array}$} \\
\hline & $\begin{array}{c}\text { Source } \\
\text { Language }\end{array}$ & Key answer & Students' answer & \\
\hline 9. & $\begin{array}{l}\text { This kind of } \\
\text { factory } \\
\text { traineeship } \\
\text { concept will } \\
\text { benefit the } \\
\text { industry in the } \\
\text { long run as } \\
\text { companies will } \\
\text { get skilled } \\
\text { labor with } \\
\text { good } \\
\text { competency }\end{array}$ & $\begin{array}{l}\text { Konsep pelatihan } \\
\text { perusahaan } \\
\text { semacam ini akan } \\
\text { menguntungkan } \\
\text { industri dalam } \\
\text { jangka panjang } \\
\text { karena } \\
\text { perusahaan akan } \\
\text { mendapatkan } \\
\text { tenaga kerja } \\
\text { terampil dengan } \\
\text { kompetensi yang } \\
\text { baik. }\end{array}$ & 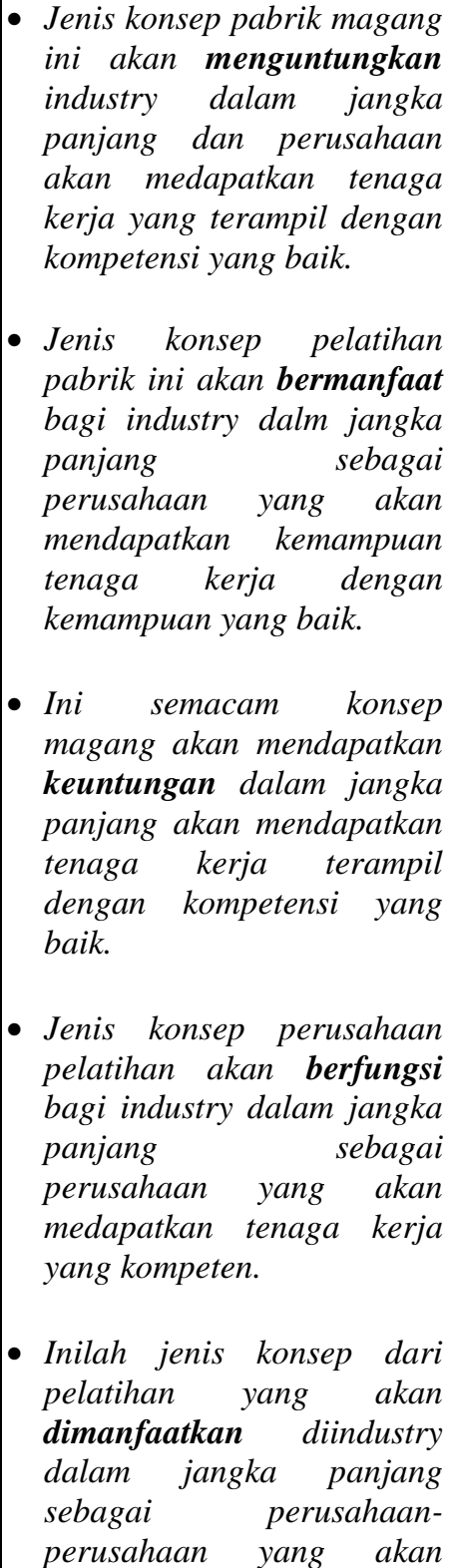 & $\begin{array}{l}-04,05,06,08 \\
09,12,13,15, \\
\text { 18, 19, 21, 22, } \\
\text { 23, 28, 30, 31, } \\
32,33,34\end{array}$ \\
\hline
\end{tabular}


Journal of English Teaching Adi Buana, Vol. 04 No. 01, April 2019

\begin{tabular}{|l|l|l|l|l|}
\hline & & $\begin{array}{l}\text { memperoleh tenaga kerja } \\
\text { dengan kompetensi yang } \\
\text { baik. }\end{array}$ & \\
\hline
\end{tabular}

Table 7: The Sample of Textual Meaning Error in English into Indonesian Translation

\begin{tabular}{|c|c|c|c|c|}
\hline \multirow[t]{2}{*}{ No } & \multirow{2}{*}{$\begin{array}{c}\text { The } \\
\text { Translation } \\
\text { Test } \\
\text { Source } \\
\text { Language } \\
\end{array}$} & \multicolumn{2}{|c|}{ The Result Test Data } & \multirow{2}{*}{\begin{tabular}{|c|}
$\begin{array}{c}\text { Identification } \\
\text { of Students }\end{array}$ \\
$\begin{array}{c}\text { Students' } \\
\text { Number }\end{array}$ \\
\end{tabular}} \\
\hline & & Key answer & Students' answer & \\
\hline 10. & $\begin{array}{l}\text { This kind of } \\
\text { factory } \\
\text { traineeship } \\
\text { concept will } \\
\text { benefit the } \\
\text { industry in the } \\
\text { long run as } \\
\text { companies will } \\
\text { get skilled } \\
\text { labor with } \\
\text { good } \\
\text { competency }\end{array}$ & $\begin{array}{l}\text { Konsep pelatihan } \\
\text { perusahaan } \\
\text { semacam ini akan } \\
\text { menguntungkan } \\
\text { industri dalam } \\
\text { jangka panjang } \\
\text { karena } \\
\text { perusahaan akan } \\
\text { mendapatkan } \\
\text { tenaga kerja } \\
\text { terampil dengan } \\
\text { kompetensi yang } \\
\text { baik. }\end{array}$ & $\begin{array}{l}\text { - Konsep pelatihan yang baik } \\
\text { dari pabrik ini akan } \\
\text { bermanfaat pada industry } \\
\text { diwaktu yang lama sebagai } \\
\text { perusahaan-perusahaan } \\
\text { yang akan mendapatkan } \\
\text { karyawan yang } \\
\text { berkemampuan dengan } \\
\text { kompetensi yang baik. } \\
\text { - Jenis konsep perusahaan } \\
\text { pelatihan akan bermanfaat } \\
\text { bagi industry dalam jangka } \\
\text { waktu panjang sebagai } \\
\text { perusahaan yang akan } \\
\text { medapatkan tenaga kerja } \\
\text { yang kompeten. } \\
\text { - Jenis konsep pelatihan } \\
\text { pabrik ini akan bermanfaat } \\
\text { di industry sepanjang } \\
\text { perusahaan akan } \\
\text { mendapatkan karyawan } \\
\text { dengan kemampuan yang } \\
\text { bagus. }\end{array}$ & $\begin{array}{l}-01,02,03,04, \\
05,08,09,12, \\
13,14,16,17, \\
19,20,21,22, \\
23,24,25,27, \\
29,33,34,35, \\
36,37 \\
-06,18,28,30, \\
32\end{array}$ \\
\hline 11. & $\begin{array}{l}\text { This kind of } \\
\text { factory } \\
\text { traineeship } \\
\text { concept will } \\
\text { benefit the } \\
\text { industry in the } \\
\text { long run as } \\
\text { companies will } \\
\text { get skilled } \\
\text { labor with } \\
\text { good } \\
\text { competency. }\end{array}$ & $\begin{array}{l}\text { Konsep pelatihan } \\
\text { perusahaan } \\
\text { semacam ini akan } \\
\text { menguntungkan } \\
\text { industri dalam } \\
\text { jangka panjang } \\
\text { karena } \\
\text { perusahaan akan } \\
\text { mendapatkan } \\
\text { tenaga kerja }\end{array}$ & $\begin{array}{l}\text { - Jenis konsep perusahaan } \\
\text { pelatihan akan berfungsi } \\
\text { bagi industry dalam jangka } \\
\text { panjang sebagai } \\
\text { perusahaan yang akan } \\
\text { medapatkan tenaga kerja } \\
\text { yang kompeten. }\end{array}$ & $\begin{array}{l}\cdot 03,04,05,06, \\
07,08,09,11, \\
12,13,19,23, \\
29,30,31,32, \\
33,34,35\end{array}$ \\
\hline
\end{tabular}


Table 8: The Sample of Textual Meaning Error in English into Indonesian Translation

\begin{tabular}{|c|c|c|c|c|}
\hline \multirow[t]{2}{*}{ No } & \multirow{2}{*}{$\begin{array}{c}\text { The } \\
\text { Translation } \\
\text { Test } \\
\text { Source } \\
\text { Language } \\
\end{array}$} & \multicolumn{2}{|c|}{ The Result Test Data } & \multirow{2}{*}{$\begin{array}{c}\begin{array}{c}\text { Identification } \\
\text { of Students }\end{array} \\
\begin{array}{c}\text { Students' } \\
\text { Number }\end{array}\end{array}$} \\
\hline & & Key answer & Students' answer & \\
\hline & & $\begin{array}{l}\text { terampil dengan } \\
\text { kompetensi yang } \\
\text { baik. }\end{array}$ & \begin{tabular}{|lr} 
- Ini semacam konsep latihan \\
kerja pabrik akan \\
menguntungkan industry \\
dalam jangka panjang \\
seperti perusahaan yang \\
akan mendapatkan \\
kemampuan tenaga kerja \\
yang baik
\end{tabular} & • 02 \\
\hline
\end{tabular}

The Frequency of Occurance

Table 9: the percentage of word class distribution error

\begin{tabular}{|l|l|l|l|l|l|}
\hline No. & Classification & Word/phrase & $\begin{array}{c}\text { Sum of } \\
\text { Errors }\end{array}$ & $\begin{array}{c}\text { Percentage } \\
(\%)\end{array}$ & Average \\
\hline 1. & Verb & Sees & 37 & 100 & 75.23 \\
& & Being invited & 12 & 32.43 & \\
& & $\begin{array}{l}\text { Getting involved } \\
\text { Reach }\end{array}$ & 18 & 48.65 & \\
& & $\begin{array}{l}\text { Enter the } \\
\text { workforce } \\
\text { Benefit }\end{array}$ & 37 & 100 & \\
& & $\begin{array}{l}\text { Developed } \\
\text { countries }\end{array}$ & 27 & 89.2 & \\
& & Interns & 36 & 72.08 & \\
\hline 2. & Noun & By the time & 28 & 97.3 & 85 \\
& & Long run & 11 & 75.68 & 52.7 \\
\hline 3. & Adverb & As & 37 & 100 & 100 \\
\hline 4. & Conjunction & & & & \\
\hline
\end{tabular}

Based on the table above, the errors of translating textual meaning in this research occur in some classes of part of speech, they are $73.43 \%$ of verb, $85 \%$ of noun, $52.7 \%$ of adverb and $100 \%$ of conjunction. From the table above, the researcher finds that the error in translating textual meaning occurs $100 \%$ of conjunction class. It is the biggest percentage of all the error which is automatically be the dominant error of textual meaning in this research. The next rank is noun class for about $85 \%$ which the most error is interns with $97.3 \%$. The third place is verb class, which is $75.23 \%$. The common error in verb class is reached and sees with $100 \%$. The last rank is adverb class with $52.7 \%$, which was $75.08 \%$ of errors by the time.

\section{DISCUSSION}

From the result of the analysis, the researcher received the data of the errors in translating textual meaning in this research occur in some classes of part of speech, they are $73.43 \%$ of verb, $85 \%$ of noun, $52.7 \%$ of adverb and $100 \%$ of conjunction. The errors in translating textual meaning occur in verb class, those are $89.2 \%$ of sees, $32.43 \%$ of being invited, $48.65 \%$ of getting involved, $100 \%$ of 
reach, $89.2 \%$ of entering the workforce and $81.08 \%$ of the benefit. While the wrong answers in translating textual meaning occur in noun class are $72.97 \%$ of developed countries and $97.3 \%$ of interns. In adverb class is $52.7 \%$, the errors are $75.68 \%$ in by the time and $29.73 \%$ in long run. The last is conjunction class that is $100 \%$. The researcher finds the answer to "what is the most dominant error make by students on textual meaning in translation English into Indonesian text?", that is $100 \%$ of as in class of speech conjunction.

From the text of translation test, there are 5 words and 6 phrases that can be identified into two categories. For the first example category is word, word "sees", based on the lexical meaning or dictionary "sees" is "melihat", however, if the word "sees" is put in the sentence "Minister sees Germany, Switzerland as models for vocational schools", it should be translated into "memandang". There are some students, who translate the word "sees" in the sentence into "menunjuk", and also there are students who translate it into "mengamati". Those are true, although the right answer should be "memandang", because there is the equivalence between "mengamati" and "memandang". So, the answer is considered right answer but it is not suitable for textual meaning.

The next example is phrase, the phrase "developed countries" based on the lexical meaning or dictionary "developed countries" is "Negara-negara berkembang", yet if the word "developed countries" is put in the sentence "Industry Minister Airlangga Hartarto has urged the country to use developed countries like Germany or Switzerland as examples in developing vocational schools as an inseparable part of the industrialization process", it should be translated into "Negara-negara maju". There are some students, who translate the word "developed countries" in the sentence into "negara-negara berkembang", perkembangan Negara-negara", and "pengembangan Negara". Those are the wrong answers because there is no equivalence between "negara-negara berkembang", "perkembangan Negara-negara", and "pengembangan Negara" with "negara-negara maju".

\section{CONCLUSION}

The study concludes that the students still face difficulties in translation, especially in textual meaning and adjustment; they are unable to translate the sentences correctly. Otherwise, the main objective of this experiment is to investigate the dominant errors made by students in translating English into Indonesian especially dealing with textual meaning. In addition, data analysis and the result of computation explained in the previous discussion, the writer draws the conclusion as follow: Through the result of the identifying textual meaning, the researcher finds that the frequency of errors made by the student in translating English into Indonesian in this research is $73.43 \%$ of a verb, $85 \%$ o a noun, $52.7 \%$ of adverb and $100 \%$ of conjunction. Based on that result, it can be concludes that the common error in translating textual meaning occurs is the conjunction class which is $100 \%$. The error is completely in one word which is as. This happens for two reasons. The first, the students translate as, but they make error in translating it. Another reason, some students do not translate it at all. 


\section{SUGGESTION}

Based on these results, researchers give a suggestion to anyone who is interested in doing research on a similar topic. The researcher suggests taking the interview as additional collecting data technique for convincing the purpose of the research. As an alternative, further research is preferable to analyze in depth possibilities of what factors are causing the student making an error in translating the text.

\section{REFERENCES}

Apriyanti, T., Wulandari, H., Safitri, M., \& Dewi, N. (2016). Translating Theory of English into Indonesian and, 2(1), 38-59.

Arikunto, S. (2006). Prosedur Penelitian : Suatu Pendekatan Praktik ( Edisi Revisi Bab IV ). Jakarta: PT. Rineka Cipta.

Ashton, E. B. (2014). The Creativity of Translation and Is Universality in the Hermeneutic Process. In Delos VI (p. 25).

Brown, H. D. (2000). Principles of Language Learning and Teaching. New York: Longman.

Cochran, M. Q. (2002). qualitative-Research-Methodology.pdf. Dipetik April Friday, 2017, dari http://d1pbog36rugm0t.cloudfront.net: http://d1pbog36rugm0t.cloudfront.net/-/media/science/research-andteaching/teaching/qualitative-research-methodology.pdf

Ellis, R., \& Gary, B. (2005). Analyzing Learner Language. UK: Oxford University Press.

Fauziati, E. (2017). Native And Target Language Influence On The Students ' Interlanguage Production: A Case Of Indonesian Efl Compositions. Indonesian Journal of Applied Linguistics, 7(1), 54-63.

Hancock, B., Ockleford, E., \& Windrigde, K. (2009). An Introduction to Qualitative Research. Nottingham: The NIHR RDS for the East Midlands.

Hatim, B., \& Munday, J. (2004). Translation: An Advanced Resource Book. USA, Canada: Psychology Press.

Hawalia, R. C. (2014). An Analysis of Textual Meaning in Translation.Lampung: UMM

Ismail, Adam; Hartono, R. (2016). Errors Made In Google Translate In The Indonesian To English Translations Of News Item Texts. Journal of English Language Teaching, 5(2), 1-6.

Manser, H. M. (2004). Oxford Learner's Pocket Dictionary. New York: Oxford University Press.

Mujiyanto, Y. (2017). The Verbal Politeness Of Interpersonal Utterances Resulted From Back-Translating Indonesian Texts Into English. Indonesian Journal of Applied Linguistics, 288-300.

Nadir Durrani, Fahim Dalvi, Hassan Sajjad, Yonatan Belinkov, P. N. (2018). What Is in a Translation Unit? Comparing Character and Subword Representations Beyond Translation. Open Review, 1-14. Retrieved from https://openreview.net/forum?id=B1x0E2C5tQ 
Notoatmodjo, S. (2005). Metode Penelitian Kesehatan. Jakarta: Rineka Cipta.

Nursalam. (2003). Konsep dan Penerapan Metodologi Penelitian Ilmu Keperawatan. Jakarta: Selemba Medika

Olk, H. (2003). Cultural Knowledge in Translation. ELT Journal, 2.

Rahayu, A. U. (2015). Differences on Language Structure between English and Indonesian. International Journal of Language, Literature and Linguistics, 1(4), 257-260. https://doi.org/10.18178/ijll1.2015.1.4.49

Richard, J., \& Schmidt, R. (2002). Longman Dictionary of Language Teaching and Applied Linguistic, 3rd Ed. London: Pearson Education Limited.

Sandelowski, M. (2000). Focus on Research Methods: What Happened to Qualitative Description? Research in Nursing \& Health.

Subandowo, D. (2017). The Language Interference In English Speaking Ability For Efl Learners.

Sugiyono. (2010). Metode Penelitian Kuantitatif Kualitatif \& RND. Bandung: Alfabeta.

.Yuliasri, I. (2016). Translation Techniques And Pragmatic Equivalence In Indonesian Translation Of Humorous Utterences In The Walt Disney's Donald Duck Comics. In International Seminar PRASASTI III: Current Research in Linguistics (pp. 409-414). Solo, Central Java: UNS. 